\title{
Hydrodynamic Flow between Two Non-Coincident Rotating Disks Embedded in Porous Media
}

\author{
Rabindranath Jana ${ }^{1}$, Mrinal Maji ${ }^{1}$, Sanatan Das ${ }^{1}$, Sovan Lal Maji ${ }^{1}$, Swapan Kumar Ghosh ${ }^{2}$ \\ ${ }^{1}$ Department of Applied Mathematics, Vidyasagar University, West Bengal, India \\ ${ }^{2}$ Department of Mathematics, Narajole Raj College, Narajole, India \\ E-mail: g_swapan2002@yahoo.com \\ Received February 28, 2011; revised April 1, 2011; accepted April 2, 2011
}

\begin{abstract}
Hydrodynamic viscous incompressible fluid flow through a porous medium between two disks rotating with same angular velocity $\Omega$ about two non-coincident axes has been studied. An exact solution of the governing equations has been obtained in a closed form. It is found that the primary velocity decreases and the secondary velocity increases with increase in porosity parameter to the left of the z-axis and the result is reversed to the right of the z-axis. It is also found that the torque on the disks increases with increase in either rotation parameter or porosity parameter. For large rotation, there exist a thin boundary layer near the disks and the thickness of this boundary layer decreases with increase in porosity parameter.
\end{abstract}

Keywords: Hydrodynamic, Non-Coincident Disks, Porous Medium, Boundary Layer Thickness, Rotation Parameter

\section{Introduction}

Hydrodynamic viscous incompressible fluid flow through a porous medium have been attracted the attention of number of researchers due to its wide applications in engineering and geophysical applications, viz. in the petroleum technology to study the movement of natural gas, oil and water through the oil reservoirs, in chemical engineering for filtration and purification process, in agriculture engineering to study the underground water resources. The viscous incompressible fluid flow due to non-coaxial rotations of a disk and a fluid at infinity has been studied by a number of researchers. The flow of a viscous incompressible fluid confined between two parallel plates rotating with the same angular velocity about non-coincident axes has been studied by Berker [1] and Abbott and Walters [2]. Erdogan [3,4] have studied the flow due to parallel disks rotating about non-coincident axes with one of the disk or both the disks oscillating in its own plane. The fluid flow between eccentric rotating disks under different environment has been studied by Ersoy [5-7]. The flow of a viscoelastic fluid between rotating disks has been studied by Rajagopal [8]. The hydromagnetic flow between two rotating disks with noncoincident parallel axes of rotation has been studied by Mohanty [9]. Kanch and Jana [10] have investigated the
Hall effects on hydromagnetic flow between two rotating disk with non-coincident parallel axes of rotation. Guria et al. [11] have studied the unsteady MHD flow between two disks with non-coincident parallel axes of rotation. Maji et al. [12] have studied the effect of rotation on unsteady MHD flow between disks, rotating with same angular velocity about two different axes. Guria et al. [13] have studied the magnetohydrodynamic flow with reference to non-coaxial rotation of a porous disk and a fluid at infinity. Effects of Hall current on unsteady MHD flow between disks with non-coincident parallel axes of rotation have been studied by Das et al. [14].

In this paper, we have studied the combined effects of rotation and porosity on the hydrodynamic flow between two disks rotating with same angular velocity about two different axes at a distant $a$ apart. In the light of our present problem it is rigorously stated that there arises symmetric motion about the mid plane between the two disks as referred to a rigid body rotation.

\section{Formulation of the Problem and Its Solution}

Let us consider the steady flow of a viscous incompressible fluid between two parallel disks, embedded in a porous medium, rotating with uniform angular velocity 
$\Omega$ about two different axes at a distance $a$ apart. We choose a system of cylindrical polar co-ordinates $(r, \theta, z)$ with the axis normal to the disks as situated symmetrically between two axes of rotation. The axis of rotation of the disk $z=h$ lies to the right and that of the disk $z=0$ lies to the left of the $z$-axis [see Figure 1].

The Navier-Stokes equation of motion is

$$
\rho(\boldsymbol{q} \cdot \nabla) \boldsymbol{q}=-\nabla p+\mu \nabla^{2} \boldsymbol{q}-\frac{\mu}{k^{*}} \boldsymbol{q}
$$

where $\boldsymbol{q}, p, \rho, \mu$ and $k^{*}$ are, respectively, the velocity vector, the fluid pressure, the density of the fluid, the fluid viscosity and the permeability of the porous medium.

The equation of continuity is

$$
\nabla \cdot \boldsymbol{q}=0 .
$$

The boundary conditions of the problem are

$$
\begin{aligned}
& u=-\frac{1}{2} \Omega a \cos \theta, v=\Omega\left(r+\frac{1}{2} a \sin \theta\right), w=0 \text { at } z=0, \\
& u=\frac{1}{2} \Omega a \cos \theta, v=\Omega\left(r-\frac{1}{2} a \sin \theta\right), w=0 \text { at } z=h .
\end{aligned}
$$

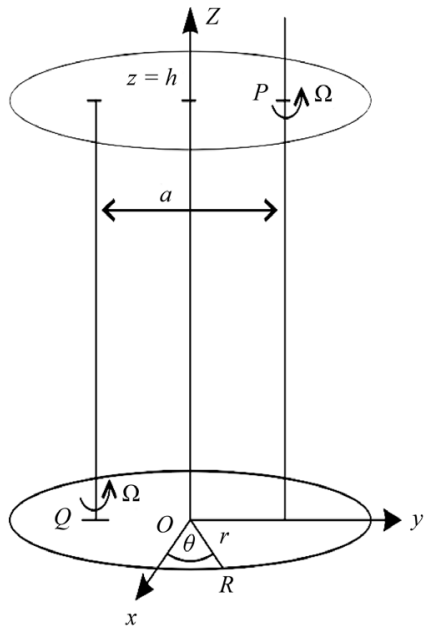

Figure 1. Geometry of the problem.

Subject to the above boundary conditions, we assume the velocity components as

$$
\begin{aligned}
& u=A(z) \cos \theta+B(z) \sin \theta, \\
& v=\Omega r+B(z) \cos \theta-A(z) \sin \theta, \\
& w=0 .
\end{aligned}
$$

On the use of (4), we have from Equation (1)

$$
\begin{array}{r}
-\Omega^{2} r-\Omega(B \cos \theta-A \sin \theta)=-\frac{1}{\rho} \frac{\partial p}{\partial r}+\frac{\mu}{\rho}\left(A^{\prime \prime} \cos \theta+B^{\prime \prime} \sin \theta\right)-\frac{\mu}{\rho k^{*}}(A \cos \theta+B \sin \theta), \\
\Omega(A \cos \theta+B \sin \theta)=-\frac{1}{\rho} \frac{\partial p}{\partial \theta}+\frac{\mu}{\rho}\left(B^{\prime \prime} \cos \theta-A^{\prime \prime} \sin \theta\right)-\frac{\mu}{\rho k^{*}}(\Omega r+B \cos \theta-A \sin \theta),
\end{array}
$$

$$
\rho g=-\frac{1}{\rho} \frac{\partial p}{\partial z},
$$

where prime refers to differentiation with respect to $z$. It

is interesting to note that Equations (5)-(7) do not contain any non-linear terms in $A(z)$ and $B(z)$.

Eliminating $p$ from Equations (5)-(7), we obtain

$$
\begin{array}{r}
-\Omega\left(B^{\prime} \cos \theta-A^{\prime} \sin \theta\right)=\frac{\mu}{\rho}\left(A^{\prime \prime \prime} \cos \theta+B^{\prime \prime \prime} \sin \theta\right)-\frac{\mu}{\rho k^{*}}\left(A^{\prime} \cos \theta+B^{\prime} \sin \theta\right), \\
\Omega\left(A^{\prime} \cos \theta+B^{\prime} \sin \theta\right)=\frac{\mu}{\rho}\left(B^{\prime \prime \prime} \cos \theta-A^{\prime \prime \prime} \sin \theta\right)-\frac{\mu}{\rho k^{*}}\left(B^{\prime} \cos \theta-A^{\prime} \sin \theta\right),
\end{array}
$$

which imply

$$
\begin{aligned}
& \frac{\mu}{\rho} \frac{\mathrm{d}^{3} A}{\mathrm{~d} z^{3}}+\Omega \frac{\mathrm{d} B}{\mathrm{~d} z}-\frac{\mu}{\rho k^{*}} \frac{\mathrm{d} A}{\mathrm{~d} z}=0, \\
& \frac{\mu}{\rho} \frac{\mathrm{d}^{3} B}{\mathrm{~d} z^{3}}-\Omega \frac{\mathrm{d} A}{\mathrm{~d} z}-\frac{\mu}{\rho k^{*}} \frac{\mathrm{d} B}{\mathrm{~d} z}=0 .
\end{aligned}
$$

The boundary conditions (3) become

$$
\begin{aligned}
& A(0)=-\frac{1}{2} \Omega a, B(0)=0, \\
& A(h)=\frac{1}{2} \Omega a, B(h)=0 .
\end{aligned}
$$

Equations (5) and (6) can be written in a dimensionless form as

$$
\begin{aligned}
& \frac{\mathrm{d}^{3} f}{\mathrm{~d} \eta^{3}}+K^{2} \frac{\mathrm{d} g}{\mathrm{~d} \eta}-\frac{1}{\sigma} \frac{\mathrm{d} f}{\mathrm{~d} \eta}=0, \\
& \frac{\mathrm{d}^{3} g}{\mathrm{~d} \eta^{3}}-K^{2} \frac{\mathrm{d} f}{\mathrm{~d} \eta}-\frac{1}{\sigma} \frac{\mathrm{d} g}{\mathrm{~d} \eta}=0,
\end{aligned}
$$

where

$$
f(\eta)=\frac{A}{a \Omega}, g(\eta)=\frac{B}{a \Omega}, \eta=\frac{z}{h}, K^{2}=\frac{\rho \Omega h^{2}}{\mu}, \sigma=\frac{k^{*}}{h^{2}} .
$$


Integrating the above Equations (13) and (14) with respect to $\eta$, we get

$$
\begin{aligned}
& \frac{\mathrm{d}^{2} f}{\mathrm{~d} \eta^{2}}+K^{2} g-\frac{1}{\sigma} f=c_{1}, \\
& \frac{\mathrm{d}^{2} g}{\mathrm{~d} \eta^{2}}-K^{2} f-\frac{1}{\sigma} g=c_{2},
\end{aligned}
$$

where $c_{1}$ and $c_{2}$ are unknown constants.

The corresponding boundary conditions (12) become

$$
\begin{aligned}
& f(0)=-\frac{1}{2}, g(0)=0, \\
& f(1)=\frac{1}{2}, g(1)=0 .
\end{aligned}
$$

Combining Equations (16) and (17), we have

$$
\frac{\mathrm{d}^{2} F}{\mathrm{~d} \eta^{2}}-\left(\frac{1}{\sigma}+\mathrm{i} K^{2}\right) F=c,
$$

where

$$
F=f+\mathrm{i} g, c=c_{1}+\mathrm{i} c_{2} \text { and } \mathrm{i}=\sqrt{-1} .
$$

The boundary conditions (18) reduce to

$$
F(0)=-\frac{1}{2}, \quad F(1)=\frac{1}{2}
$$

In the case of a symmetric flow, we may choose the constant $c=0$ and the Equation (19) becomes

$$
\frac{\mathrm{d}^{2} F}{\mathrm{~d} \eta^{2}}-\left(\frac{1}{\sigma}+\mathrm{i} K^{2}\right) F=0,
$$

The solution of the Equation (22) subject to the boundary conditions (21) can be obtained in the following form

$$
F(\eta)=\frac{1}{2}\left[\frac{\sinh (\alpha+\mathrm{i} \beta) \eta}{\sinh (\alpha+\mathrm{i} \beta)}-\frac{\sinh (\alpha+\mathrm{i} \beta)(1-\eta)}{\sinh (\alpha+\mathrm{i} \beta)}\right],
$$

where

$$
\begin{aligned}
& \alpha=\frac{1}{\sqrt{2}}\left[\left(\frac{1}{\sigma^{2}}+K^{4}\right)^{\frac{1}{2}}+\frac{1}{\sigma}\right]^{\frac{1}{2}}, \\
& \beta=\frac{1}{\sqrt{2}}\left[\left(\frac{1}{\sigma^{2}}+K^{4}\right)^{\frac{1}{2}}-\frac{1}{\sigma}\right]^{\frac{1}{2}} .
\end{aligned}
$$

Using (20) with the separating of real and imaginary parts, we have

$$
\begin{aligned}
f(\eta)= & \frac{1}{(\cosh 2 \alpha-\cos 2 \beta)}[\{\sinh \alpha \eta \cos \beta \eta+\sinh (\eta-1) \alpha \cos (\eta-1) \beta\} \sinh \alpha \cos \beta \\
& +\{\cosh \alpha \eta \sin \beta \eta+\cosh (\eta-1) \alpha \sin (\eta-1) \beta\} \cosh \alpha \sin \beta], \\
g(\eta)= & \frac{1}{(\cosh 2 \alpha-\cos 2 \beta)}[\{\cosh \alpha \eta \sin \beta \eta+\cosh (\eta-1) \alpha \sin (\eta-1) \beta\} \sinh \alpha \cos \beta \\
& -\{\sinh \alpha \eta \cos \beta \eta+\sinh (\eta-1) \alpha \cos (\eta-1) \beta\} \cosh \alpha \sin \beta] .
\end{aligned}
$$

If $\sigma=\infty$, i.e. in the absence of a porosity of the medium, the above equations reduce to

$$
\begin{aligned}
f(\eta)= & \frac{1}{(\cosh 2 K-\cos 2 K)}[\{\sinh K \eta \cos K \eta+\sinh (\eta-1) K \cos (\eta-1) K\} \sinh K \cos K \\
& +\{\cosh K \eta \sin K \eta+\cosh (\eta-1) K \sin (\eta-1) K\} \cosh K \sin K], \\
g(\eta)= & \frac{1}{(\cosh 2 K-\cos 2 K)}[\{\cosh K \eta \sin K \eta+\cosh (\eta-1) K \sin (\eta-1) K\} \sinh K \cos K \\
& -\{\sinh K \eta \cos K \eta+\sinh (\eta-1) K \cos (\eta-1) K\} \cosh K \sin K] .
\end{aligned}
$$

which are identical with Equations (21) and (22) as the result obtained by Abbott and Walters[10].

\section{Results and Discussions}

To study the effects of rotation and porosity on the flow between two disks embedded in porous medium with non-coincident parallel axes of rotation, the dimensionless primary velocity $f$ and secondary velocity $g$ are depicted graphically against $\eta$ for various values of rotation parameter $K^{2}$ and porosity parameter $\sigma$ in Figures 2-3. Figure 2 demonstrates that the velocities $f$ and $g$ increase with an increase in $K^{2}$ to the left of the $\mathrm{z}$-axis while to the right of the $\mathrm{z}$-axis they decrease with 


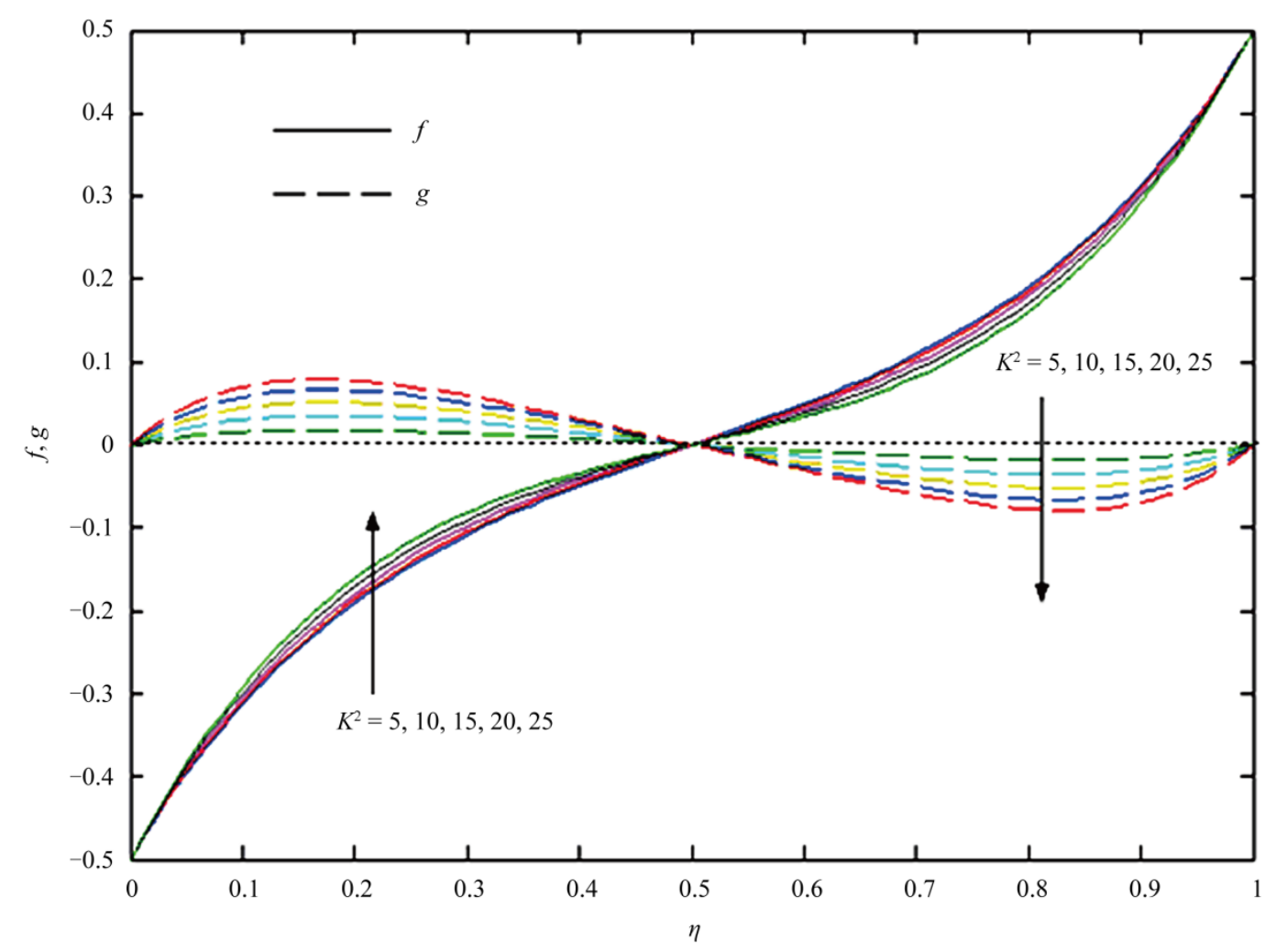

Figure 2. Variations of primary velocity $f$ and secondary velocity $g$ for $\sigma=0.2$.

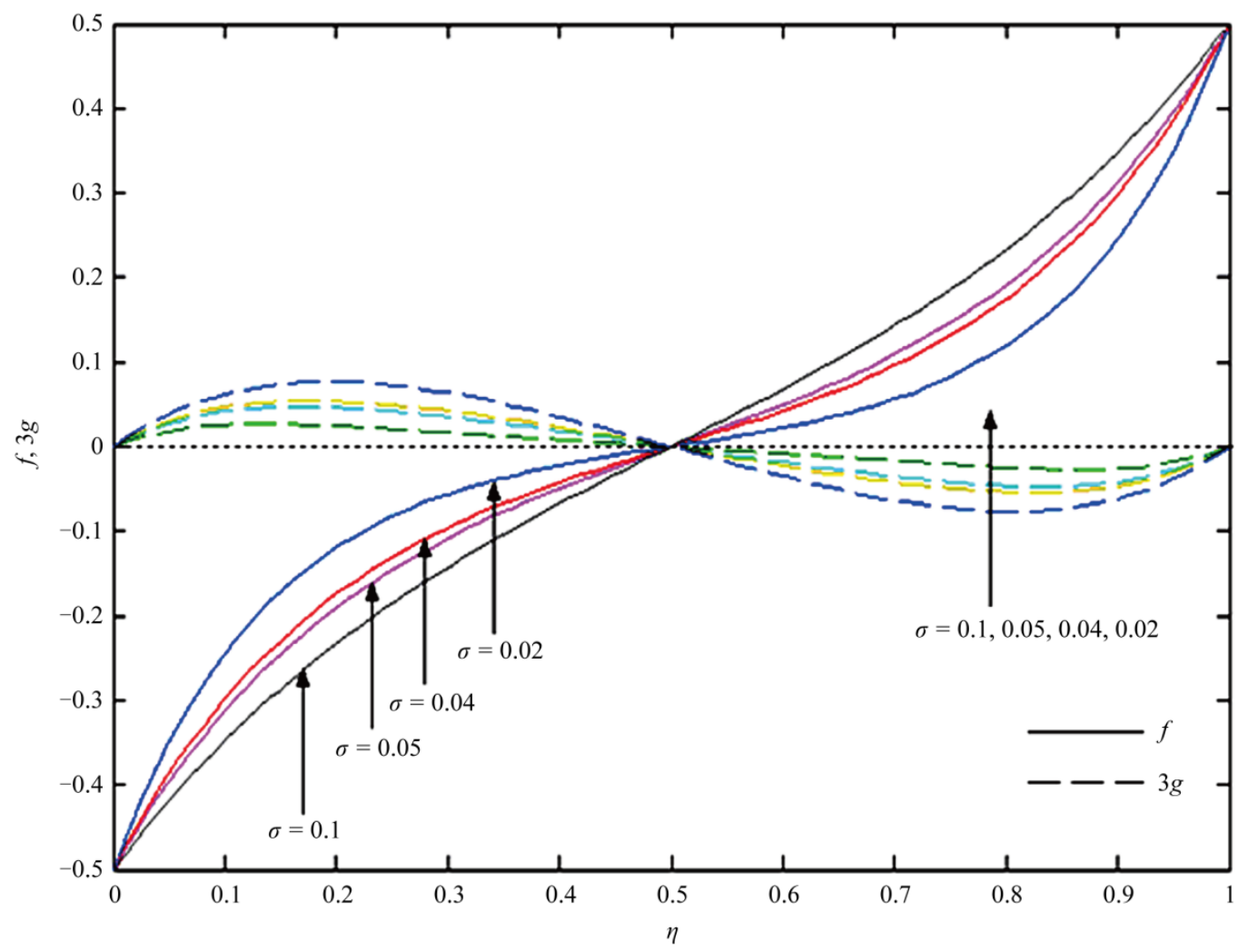

Figure 3. Variations of primary velocity $f$ and secondary velocity $g$ for $K^{2}=5$. 
increase in $K^{2}$. Figure 3 shows that the primary velocity $f$ decreases with an increase in $\sigma$ to left of the $\mathrm{z}$-axis while it increases with an increase in $\sigma$ to the right of the z-axis. On the other hand, the secondary velocity $g$ increases with an increase in $\sigma$ to the left of the z-axis while it becomes reversed to right of the $\mathrm{z}$-axis.

The non-dimensional shear stresses due to the primary and the secondary flows at the disks $\eta=0$ and $\eta=1$ are given by

$$
\begin{aligned}
& {\left[f^{\prime}(\eta)\right]_{(0,1)}=\left(\frac{\cosh \alpha+\cos \beta}{\cosh 2 \alpha-\cos 2 \beta}\right)(\alpha \sinh \alpha+\beta \sin \beta),} \\
& {\left[g^{\prime}(\eta)\right]_{(0,1)}=\left(\frac{\cosh \alpha+\cos \beta}{\cosh 2 \alpha-\cos 2 \beta}\right)(\beta \sinh \alpha-\alpha \sin \beta) .}
\end{aligned}
$$

The torque exerted by the fluid to overcome the transverse shearing stress on a disk of radius $b$, such as

$$
\operatorname{Torque}\left(\tau_{0}\right) \equiv 2 \int_{0}^{b} \int_{0}^{\pi} \tau_{z \theta} r^{2} \mathrm{~d} r \mathrm{~d} \theta=-\frac{1}{3} \frac{\mu \Omega a b^{3}}{h}\left(\frac{\cosh \alpha+\cos \beta}{\cosh 2 \alpha-\cos 2 \beta}\right)(\alpha \sinh \alpha+\beta \sin \beta) \text {. }
$$

The values of the torque $\tau_{0}\left(=\frac{1}{3} \frac{\mu \Omega a b^{3}}{h}\right)$ at the disks are shown in Figure 4 for different values of $\sigma$ against $K^{2}$. It is seen from Figure 4 that the torque $\left(\tau_{0}\right)$ on the disks increases with an increase in either $\sigma$ or $K^{2}$.

We shall discuss some special cases of interest:

Case 1: When $\sigma \gg 1$ and $K^{2} \ll 1$. In this case, we have from Equation (23), on separating into a real and imaginary parts

$$
\begin{gathered}
f(\eta)=\frac{1}{2}\left[(2 \eta-1)+\frac{1}{6 \sigma}\left(2 \eta^{3}-3 \eta^{2}+\eta\right)+\frac{1}{360}\left(\frac{1}{\sigma^{2}}-K^{4}\right)\left(6 \eta^{5}-15 \eta^{4}+10 \eta^{3}-\eta\right)+\cdots,\right. \\
g(\eta)=\frac{K^{2}}{2}\left[\frac{1}{6}\left(2 \eta^{3}-3 \eta^{2}+\eta\right)+\frac{1}{180 \sigma}\left(6 \eta^{5}-15 \eta^{4}+10 \eta^{3}-\eta\right)+\cdots\right]
\end{gathered}
$$

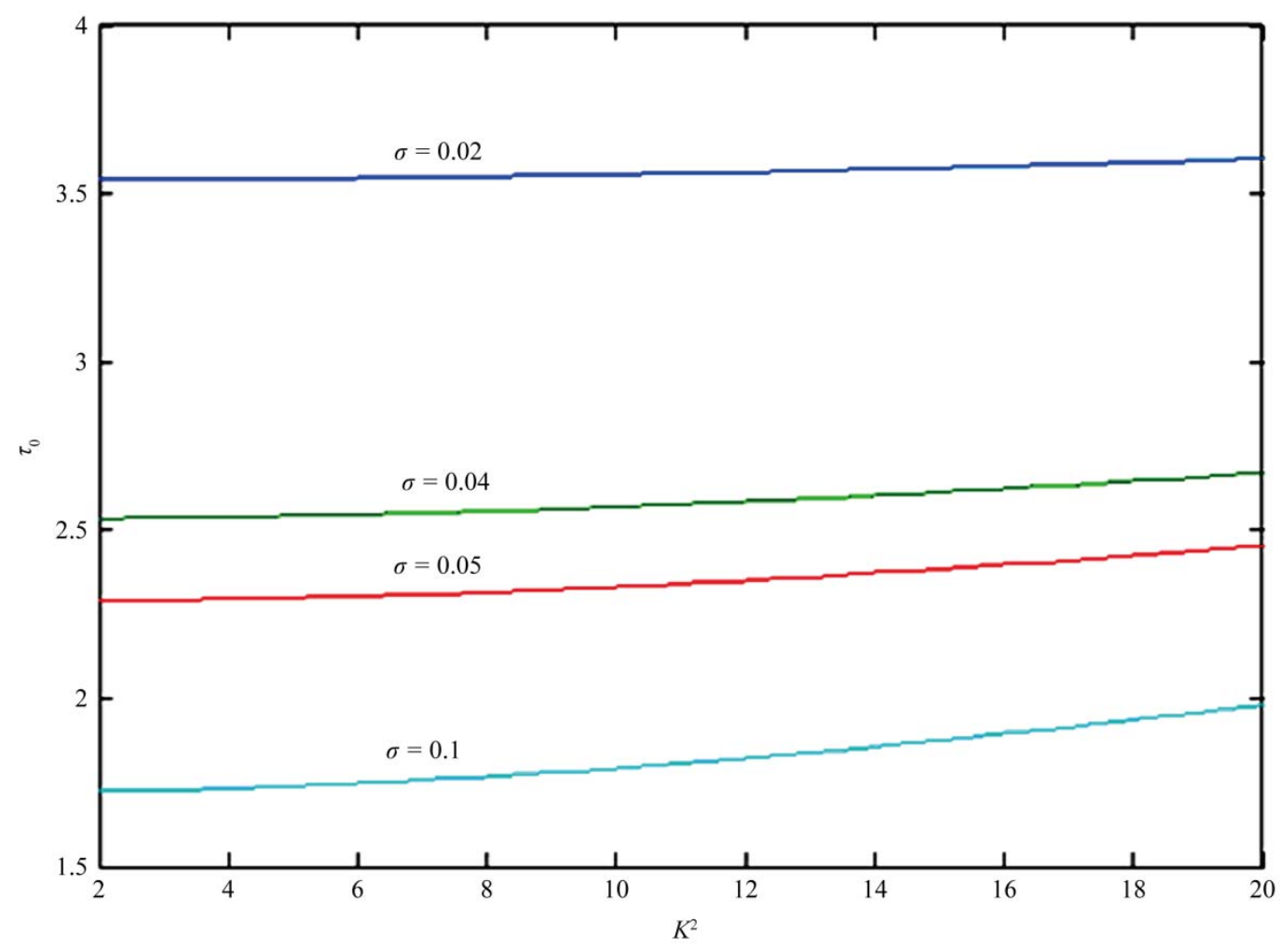

Figure 4. Variations of torque $\tau_{0}$. 
It is seen from the Equation (32) that the effects of rotation parameter $K^{2}$ on the velocity distribution $f(\eta)$ is important only when we consider the order of $O\left(K^{4}\right)$.

Case 2: For large rotation of the disks i.e. for $K^{2} \gg 1$ and $\sigma \gg 1$, we get from Equation (23) on separating into a real and imaginary parts.

$$
\begin{aligned}
& f(\eta)=\frac{1}{2}\left[\mathrm{e}^{-\alpha(1-\eta)} \cos \beta(1-\eta)-\mathrm{e}^{-\alpha \eta} \cos \beta \eta\right], \\
& g(\eta)=\frac{1}{2}\left[-\mathrm{e}^{-\alpha(1-\eta)} \sin \beta(1-\eta)+\mathrm{e}^{-\alpha \eta} \sin \beta \eta\right]
\end{aligned}
$$

where

$$
\alpha, \beta=\frac{K}{\sqrt{2}}\left(1 \pm \frac{1}{2 K^{2} \sigma}\right) .
$$

The Equations (34) and (35) show the existence of the single-deck boundary layer of thickness of the order $\mathrm{O}(1 / \alpha)$, where $\alpha$ is given by (36). This boundary layer thickness decreases with an increase in either rotation parameter $K^{2}$ or porosity parameter $\sigma$, since $\alpha$ increases with an increase in either $K^{2}$ or $\sigma$.

Case 3: When $\sigma \ll 1$ and $K^{2} \ll 1$. In this case, we have from Equation (23), on separating into a real and imaginary parts

$$
\begin{gathered}
f(\eta)=\frac{1}{2}\left[\mathrm{e}^{-\frac{1}{\sqrt{\sigma}}(1-\eta)}-\mathrm{e}^{-\frac{1}{\sqrt{\sigma}} \eta}\right], \\
g(\eta)=0 .
\end{gathered}
$$

Equation (37) demonstrates the existence of the single-deck boundary layer of thickness of the order $\mathrm{O}(\sigma)$. This boundary layer thickness increases with an increase in porosity parameter $\sigma$. It is interesting to note that for small value of $\sigma$, the boundary layer thickness is independent of rotation parameter $K^{2}$. Further, for small values of $\sigma$, the secondary velocity vanishes in the azimuthal direction.

\section{Case 4: Single rotating disk}

In the limit $h \rightarrow \infty$, that is, when the disk at $\eta=1$ is placed at an infinite distance, then the solution (23) yields, on using (20)

$$
\begin{gathered}
f(\eta)=-\frac{1}{2} \mathrm{e}^{-\alpha \eta} \cos \beta \eta, \\
g(\eta)=\frac{1}{2} \mathrm{e}^{-\alpha \eta} \sin \beta \eta,
\end{gathered}
$$

where

$$
\begin{aligned}
& \alpha=\frac{1}{\sqrt{2}}\left[\left(\frac{1}{\sigma^{2}}+1\right)^{\frac{1}{2}}+\frac{1}{\sigma}\right]^{\frac{1}{2}}, \\
& \beta=\frac{1}{\sqrt{2}}\left[\left(\frac{1}{\sigma^{2}}+1\right)^{\frac{1}{2}}-\frac{1}{\sigma}\right]^{\frac{1}{2}}, \sigma=\frac{k^{\prime} \Omega}{v}, \eta=\sqrt{\frac{\Omega}{v}} z .
\end{aligned}
$$

The Equations (39) and (40) show that there exist a thin boundary layer near the disk $\eta=0$. The thickness of this boundary layer is of order $\mathrm{O}(1 / \alpha)$ where $\alpha$ is given by (41). This boundary layer thickness increases with an increase in $\sigma$, since $\alpha$ decreases with an increase in $\sigma$. It is interesting to note that in the limit as $h \rightarrow \infty$, we arrived the result of the hydrodynamic viscous flow due to non-coincident rotations of a disk and a fluid at infinity embedded in porous medium.

\section{Conclusions}

Hydrodynamic viscous incompressible fluid flow between two non-coincident rotating disks embedded in porous media is studied. An exact solution of the governing equations has been obtained in a closed form. It is found that the primary velocity $f$ decreases and the secondary velocity $g$ increases with increase in porosity parameter to the left of the z-axis and the result is reversed to the right of the $\mathrm{z}$-axis. It is also found that the torque on the disks increases with increase in either rotation parameter or porosity parameter. In the limit of $h \rightarrow \infty$, we derived the result of hydrodynamic flow of a viscous fluid due to non-coaxial rotations of a disk and a fluid at infinity embedded in a porous medium.

\section{References}

[1] R. Berker, "Hand Book of Fluid Dynamics,” Vol. VIII/3, Springer, Berlin, 1963.

[2] T. N. G. Abbott and K. Walters, "Rheometrical Flow Systems, Part-2. Theory for Orthogonal Rheometer, Including an Exact Solution of the Navier-Stokes Equations,” Journal of Fluid Mechanics, Vol. 40, 1970, pp. 205-213.doi:10.1017/S0022112070000125

[3] M. E. Erdogan, "Unsteady Flow between Eccentric Rotating Disks Executing Non-Torsional Oscollations," International Journal of Non-Linear Mechanics, Vol. 35, No. 4, 2000, pp. 691-699. doi:10.1016/S0020-7462(99)00051-7

[4] M. E. Erdogan, "Unsteady Viscous Flow between Eccentric Rotating Disks," International Journal of Non-Linear Mechanics, Vol. 30, No. 5, 1995, pp. 711-717. doi:10.1016/0020-7462(95)00030-R

[5] H. V. Ersoy, "Unsteady Flow Due to Sudden Pull of Eccentric Rotating Disks,” International Journal of Engi- 
neering Science, Vol. 39, No. 3, 2001, pp. 343-354. doi:10.1016/S0020-7225(00)00040-9

[6] H. V. Ersoy, "Unsteady Flow Due to Concentric Rotation of Eccentric Rotating Disks,” Meccanica, Vol. 38, No. 3, 2003, pp. 325-334. doi:10.1023/A:1023374214783

[7] H. V. Ersoy, "MHD Flow of an Oldroyd-B Fluid between Eccentric Rotating Disks,” International Journal of Engineering Science, Vol. 37, No. 15, 1999, pp. 1973-1984. doi:10.1016/S0020-7225(99)00010-5

[8] K. R. Rajagopal, "Flow of Viscoelastic Fluids between Rotating Disks," Theoretical and Computational Fluid Dynamics, Vol. 3, No. 4, 1992, pp. 185-206. doi:10.1007/BF00417912

[9] H. K. Mohanty, "Hydromagnetic Flow between Two Rotating Disks with Non-Coincident Parallel Axes of Rotation," Physics of Fluids, Vol. 15, No. 8, 1972, pp. 1456-1458. doi:10.1063/1.1694107

[10] A. K. Kanch and R. N. Jana, "Hall Effects on Hydromagnetic Flow Between Two Disks with Non-Coincident Parallel Axes of Rotation,” Revue Roumaine des Sciences
Techniques-Série de Mécanique Appliquée, Vol. 37, No. 4, 1992, pp. 379- 385.

[11] M. Guria, R. N. Jana and S. K. Ghosh, "Unsteady MHD Flow Between Two Disks with Non-Coincident Parallel Axes of Rotation," International Journal of Fluid Mechanics Research, Vol. 34, No. 5, 2007, pp. 425-433. doi:10.1615/InterJFluidMechRes.v34.i5.30

[12] S. L. Maji, N. Ghara, R. N. Jana and S. Das, "Unsteady MHD Flow Between two Eccentric Rotating Disks," Journal of Physical Sciences, Vol. 13, 2009, pp. 87-96.

[13] M. Guria, B. K. Das, R. N. Jana and S. K. Ghosh, "Magnetohydrodynamic Flow With Reference to Non-Coaxial Rotation of a Porous Disk and a Fluid at Infinity,” International Journal of Dynamics of Fluids, Vol. 7, No. 1, 2011, pp. 25-34.

[14] S. Das, S. L. Maji, M. Guria and R. N. Jana, "Hall Effects on Unsteady MHD Flow Between two Disks with Non-Coincident Parallel Axes of Rotation,” International Journal of Applied Mechanics and Engineering, Vol. 15, No. 1, 2010, pp. 5-18. 